\title{
Volatile organic compounds predict response to both low FODMAP diet and probiotics in irritable bowel syndrome: a randomised controlled trial
}

\author{
M. Rossi*1, R. Aggio*2, H. Staudacher, M. Lomer, J.O. Lindsay, P. Irving, C. Probert** and \\ K. Whelan**1 \\ ${ }^{1}$ Diabetes and Nutritional Sciences, King's College London, SE1 9NH, \\ ${ }^{2}$ Guy's and St Thomas' NHS Foundation Trust, SE1 7EH, \\ ${ }^{3}$ University of Liverpool, L69 $3 B X$ and \\ ${ }^{4}$ Bart's Health NHS Trust, E1 2ES
}

Dietary intervention is an effective therapy for irritable bowel syndrome (IBS), with restriction of fermentable carbohydrates (low FODMAP diet, LFD) and microbial therapies such as probiotics showing efficacy in clinical trials ${ }^{(1)}$. The aim of this study was to investigate whether a non-invasive diagnostic model using faecal volatile organic compounds (VOCs) in IBS could be used to identify (i) what factors at baseline are associated with response to dietary interventions (predict response); and (ii) what factors at end of treatment are associated with response (explore potential mechanisms underpinning response through changes in VOCs).

Adults fulfilling Rome III criteria for IBS were screened for inclusion. Eligible patients were recruited to a $2 \times 2$ factorial randomized controlled trial and were randomized to LFD or placebo dietary advice (sham diet) and to multi-strain probiotic (VSL\#3) or placebo for 4 weeks. Response to either intervention was defined as a reduction of $\geq 50$ points for the validated IBS symptom scoring system (IBS-SSS) between baseline and end of dietary intervention (follow-up). Faecal samples were collected at baseline and follow-up. VOCs were analysed by a gas chromatography - sensor device (Odoreader). VOC profiles were processed using an in-house pipeline involving wavelet transformation followed by feature selection based on random forest ${ }^{(2)}$. Using the selected features, a partial least squares (PLS) classifier was constructed to classify VOC profiles from responders and non-responders and accuracies determined using 10-fold cross-validation.

A total of 95 patients completed the study of whom $93(63$ females, $68 \%)$ provided sufficient sample for analysis. More patients responded to LFD $(37 / 46,80 \%)$ compared to sham $(21 / 47,45 \%)(\mathrm{p}<0.001)$ but there was no difference between probiotic $(31 / 49,63 \%)$ and placebo $(27 / 44,61 \%)(\mathrm{p}=0.850)$. The PCA identified (i) 15 compounds at baseline that explained $25 \%$ of variation in response to the LFD (PLS mean accuracy of $97 \%$; CI 96-99\%, Figure 1) and 10 compounds that explained $30 \%$ of variation in response to the probiotics (PLS mean accuracy of $88 \%$; CI 86-92\%, Figure 2); and (ii) 9 compounds at end of treatment that explained $31 \%$ of response to LFD (PLS mean accuracy of $96 \%$; CI 93-98\%) and 11 compounds that explained $27 \%$ of variation in response to the probiotics (PLS mean accuracy of $91 \%$; CI $88-94 \%$ ).

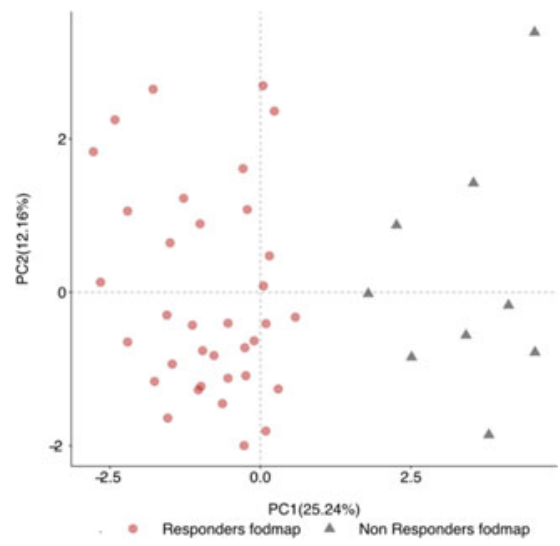

Fig. 1. Principal Component Analysis predicting response to the low FODMAP diet

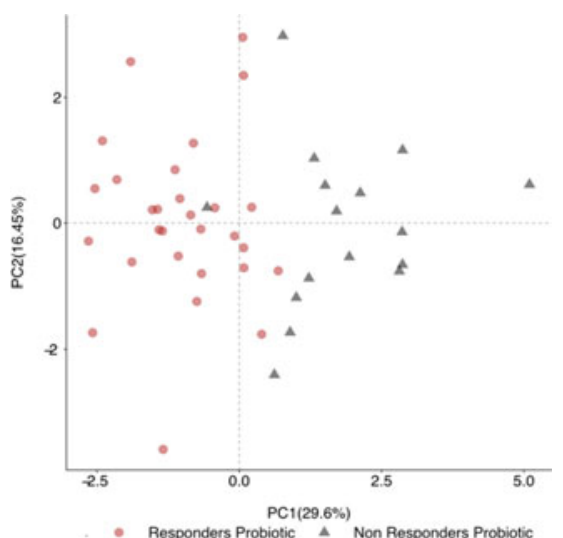

Fig. 2. Principal Component Analysis predicting response to probiotic supplementation

Faecal VOC profiling is a low cost, non-invasive tool that may help predict response to dietary intervention in IBS patients as well as shedding light on mechanisms underpinning response. Clinical trials using this algorithm are needed, and if successful, will pave the way for personalised treatment plans in IBS.

1. Staudacher HM, Irving PM, Lomer MC et al. (2014) Nat Rev Gastroenterol Hepatol 11, 256-266.

2. Aggio RM, White P, Jayasena H et al. (2017) Aliment Pharmacol Ther 45, 82-90.

*Authors contributed equal parts 